\title{
Summary of the Conference on Electronic Density of States*1
}

\author{
H. Ehrenreich \\ Division of Engineering and Applied Physics, Harvard University, Cambridge, Massachusetts
}

(October 10, 1969)

It is very difficult to summarize a conference such as this, involving as it did a many splendored array of topics, both experimental and theoretical, expounded in no less than ninety papers. The organization of a conference of such size is ordinarily impossible without resort to simultaneous sessions. When there are simultaneous sessions, of course, it is easier for a single summarizer because he can only be at one place at one time and therefore can be excused for failing to do justice to half of the papers. It is also easier for the audience because if you permit people to resonate between sessions, you also allow them to become trapped in the halls, advertently or inadvertently as the case may be. However, through the devilish cleverness of the organizers of the present conference, a goodly fraction of the papers were delivered by a rapporteur. Accordingly, the entire audience, including the summarizer were exposed to everything during a period of three and a half hard-working and elaborately organized days. Furthermore, I lose my excuse for having overlooked, as I undoubtedly did, some of the important new developments presented or presaged here.

As it is, in the time of thirty-two minutes that have been allotted to me it is, of course, impossible to mention even a representative fraction of the contributions. Indeed, even the various areas discussed here can only be sketched in broad outlines. Fortunately my task is considerably eased by the various excellent review lectures and rapporteur summaries that punctuated the conference. In order to avoid the risk of offending a few, I have decided instead to offend everybody by not mentioning names in this talk, except when referring to work which was not explicitly reported at this conference which is appropriately referenced.

\footnotetext{
*An invited paper presented at the 3d Materials Research Symposium, Electronic Density of States, November 3-6, 1969, Gaithersburg, Md.

' Supported in part by Grant No. GP-8019 of the National Science Foundation and the Advanced Research Projects Agency.
}

At the outset, let me thank the organizers on behalf of everyone attending it for providing us with an outstanding scientific program that clearly focussed on the principal questions which those of us who are grappling with the electronic structure of condensed matter are facing. During the time when we were not riding buses or listening to papers, there was also a most pleasant social program, and mercifully, a few hours for sleep.

I would regard the title of the present symposium, "Electronic Density of States" a leitmotif rather than an idee fixe because the subject matter presented at the conference in fact was far more general than might be implied by the title. Since the density of states is very influential in the determination of many basic physical properties, it provides an excellent focal point for presenting some of the more recent developments in the electronic properties of condensed matter.

More importantly (and to my view this is one of the chief motivations of this conference) the density of states is a convenient central quantity for confronting theory and experiment even though, unfortunately, it never seems to be measured directly by any experiment. In the jargon of the modern theorist one might phrase this difficulty in the following way. The density of states is proportional to the imaginary part of the single particle Green's function, whereas many experiments determine a response function, which involves Green's functions of two or more particles. When suitable approximations are made, however, the state density enters in a fairly direct way into the theoretical interpretation of all of the various kinds of measurement described at this conference.

The list of techniques available to the solid state physicist, which was extensively sampled here, is truly impressive and stands in contrast to the much more limited variety available to our colleagues in the elementary particle field. We heard about optical absorp- 
tion and reflectance, x-ray spectroscopy, photoemission, Fermi surface experiments, tunneling, measurements of the electronic specific heat, magnetic susceptibility, superconducting critical fields, and transport properties, positron annihilation, Compton scattering, ion neutralization spectroscopy among others, and how some of these are influenced by pressure, strain, and temperature.

The preceding list reflects the fact that photons continue to be one of the favorite probes for studying the microscopic properties of matter. It is therefore tempting to use the 1965 Conference in Paris on the Optical Properties and Electronic Structures of Metals and Alloys [1] which dealt with the same class of materials as the present conference and similar ideas concerning the interpretations of experiments as a fiducial mark to give us some indication of what we have learned about metals and alloys during the interim.

Pippard, in his summary of that conference, remarked on the extraordinary number of times the audience was shown the $\mathrm{Cu}$ band structure. Since then the variety of band calculations, and, in particular the kinds of materials considered has proliferated greatly. $\mathrm{Pd}$ has received a great deal of recent attention largely as a result of excellent high field susceptibility and photoemission measurements. Other examples discussed here involved more exotic materials such as $\mathrm{AuAl}_{2}, \mathrm{EuO}$ and $\mathrm{GeO}_{2}$. Evidently the machinery for doing such calculations on ordered alloys containing several atoms per unit cell, some of which are sufficiently heavy that relativistic effects become important, is now available at several laboratories in a readily usable form. However, as in all band calculations, even if one accepts the Hartree-Fock approximation, the result obtained is only as good as the potential that is used as input information. In metallic alloys one might expect some charge transfer among the atoms belonging to a single unit cell. In my view this possibility has not yet received adequate attention.

For example, $\mathrm{AuAl}_{2}$ has the $\mathrm{CaF}_{2}$ structures and is one of the few metals in which a Raman frequency has been observed [2]. Remarkably, its magnitude is similar to that of $\mathrm{CaF}_{2}$. One might ask whether the very similar stiffness of the optical frequencies in these materials is purely an accident or whether there could be enough charge transfer among the atoms in the cell to result in appreciable ionic character. I realize that I am undoubtedly not saying anything that is not already familiar to band theorists. However, experimentalists should be warned that the construction of alloy potentials, even for ordered systems, is still a problem that requires attention.
Indeed, the simpler problem of calculating band structures for monatomic metals on the single particle picture is still controversial. As we saw in connection with several of the contributions and much of the spirited discussion that followed them, we still don't understand clearly how, when, or why to localize the exchange interaction. To date no sufficient theoretical reason has been advanced for preferring either the Slater or the Gaspar-Kohn-Sham versions of this potential. Proponents of either point of view, or those favoring intermediate values of the coefficients at present usually support their position by comparison with experiment rather than basic theoretical arguments.

In this same connection we might note the debate following the introductory lecture concerning the relative merits of first principles and pseudo- or model-potential band calculations. The essential point made in that lecture, in my view, is that "pseudism" is important because it provides elementary insight into the meaning of the results of the elaborate machine computations. The two approaches, in fact, are complementary and the proponents of each have a genuine need for, and indeed ideally should merge with the other.

There is no doubt whatsoever that band theory has done sufficiently well that it is worth using its results to calculate the density of states accurately. This is a difficult numerical problem, particularly if one resignedly accepts spending ceilings that curtail the amount of available computer time, for one needs to know the energy at many millions of points in the Brillouin zone in order to construct adequate histograms that yield all the fine detail in the density of states that is often necessary to interpret experimental information reliably. We saw several examples of advances in performing such calculations more economically at this conference. The QUAD scheme is one of these. Another, which I will call the IBM scheme, not after the machine but the workers, is similar to QUAD in its ability to generate very detailed $\mathrm{E}(\mathrm{k})$ curves, but it avoids the use of histograms. All these result in very finely grained structure in the state density.

In this same connection, I think the importance of learning how to sum functions of $k$ over constant energy surfaces in the Brillouin zone efficiently and reliably needs emphasis. This is important not only for calculating the state density, but also for computing Green's functions that are central to the solution of alloy band problems, frequency dependent dielectric functions that can be compared with optical data, susceptibilities, and many other quantities. While many theorists may think such problems as insufficiently dignified, I would, nevertheless, stress that their solution is impor- 
tant if one ever expects to confront theory and experiment realistically on a more complete basis for more complicated systems.

At the same time let me temper this call to computer and numerical analysis handbooks by reminding you of the obvious fact stressed by many speakers that band calculations are single particle descriptions involving electrons or holes as ideal quasi-particles which interact with a self-consistent field that is in practice determined more or less self-consistently. However, due to electron interactions including those involving phonons, real quasi-particles acquire finite lifetimes, except right at the Fermi surface. Some of the papers presented here reflected the fact that methods of taking quasi-particle effects into account more systematically in band calculations are now being developed. I would look towards greater exploitation of such techniques in the band calculations of the near future. It is important to remember that when we speak of dressing effects, say, due to electron-phonon interactions, we ought to be dressing the right bare object, namely the correctly calculated quasi-particle appropriate to the stationary lattice.

One should again be reminded of the fact that very few if any of the experiments discussed at this meeting correspond to creation of just one quasi-particle near the Fermi surface. This fact was also stressed already at the Paris Conference. Optical experiments, for example, correspond to the creation of two quasi-particles and ion neutralization measurements to three. These may interact with each other as well as with the other particles in the system. This was illustrated in the discussion of the various types of phenomena that can occur in x-ray emission, which lead to the conclusion that the observed spectrum of the valence band may bear less resemblance than one would hope to what is calculated from band theory. In addition to the long familiar Landsberg or Auger tails that smear out the lower valence band edge, there are recently predicted elementary excitations such as the plasmaron and other broad structures that also result from interactions with plasmons. Another effect that was reported on here results from exciton type interactions between electrons near the Fermi surface and the core holes with which they combine in an x-ray emission process. These may strongly affect the transition rate and lead to substantial enhancement or dimunition of the observed intensity near the Fermi surface. As has been pointed out to us, these effects must be quantitatively understood before information concerning band structure can be reliably extracted from such experiments. As a result of particle interaction effects, the informa- tion supplied, even what would in a simple minded view correspond to the same theoretical quantity, often differs from experiment to experiment. The Fermi surface effective mass determined from electronic specific heat and Pauli susceptibility measurements is an example of this.

Even within a single particle framework, the state density function is only characteristic of a particular type of experiment. For example, in x-ray emission experiments, optical selection rules pick out only those components of the valence band state density having appropriate symmetries with respect to the core hole. We saw that this fact has particular utility in providing insight into the character of the wave function overlap and hybridization among different components in both ordered and disordered alloys. Since the core hole is localized in a given atomic site, the study of say the $\mathrm{Al}-\mathrm{L}_{2,3}$ emission spectrum in systems such as $\mathrm{AuAl}_{2}$ and others discussed here, provide an indication of the amount of $d$ wave function in these systems located on the $\mathrm{Al}$ sites.

As another example, we might mention the k-conversation rule entering interband optical processes which implies that the state density appearing in theoretical expressions for the optical constants is the so-called "joint density of states." This seemingly innocuous fact has led to a spirited controversy in connection with the interpretation of photoemission experiments which was already in full bloom at the Paris Conference. As you all know by now, there are two schools of thought whose proponents we might call the k-conservationists and the k-nonconservationists. The latter group has maintained, on the basis of a considerable body of experimental evidence, that particularly in materials having narrow bands such as the noble and transition metals, the energy distribution of the photoemitted electrons should directly reflect the structure in the density of states pertaining to these bands. The k-conservationists on the other hand have asked, "Why should this conservation law be violated?" Indeed, one of the papers, which represents the first attempt at the formulation of a systematic theory of the photoelectric effect in solids, points to ways in which this might come about.

Several of the other contributions point to progress towards a reconciliation of these viewpoints. For example, we have heard in connection with $\mathrm{Cu}$ that a direct transitions analysis using constant matrix elements accounts quite well for the observed energy distribution. Similar conclusions have been reached on the basis of very detailed calculations for $\mathrm{Pd}$. The essential point, which was emphasized by both camps, is that the strongest peak in the joint density of states coincide with 
peaks in the calculated state density, particularly in the case of narrow valence bands.

While in many cases the photoemission technique is a very useful tool, this may not be the case universally. It was suggested, for example, that it is less successful in providing information concerning $f$ states in the $\mathrm{Eu}$ chalcogenides and rare earth metals since these states are seen to give rise to abnormally low quantum yield relative to, for example, p states. It is also clear that the variation of optical matrix elements with energy and selection rules, which can also lead to structure in the observed spectra, needs further attention because in many calculations this matrix element is still regarded to be a constant.

Before leaving the subject of optical properties of crystal, two other points are worth making. Despite the fact that one learns only about the joint density of states in such experiments, it is, in fact, possible to derive the conventional state density from optical data by a more circuitous route. The usefulness of differential reflectance techniques is now well established and was illustrated in several of the contributions presented here which even extended to the x-ray case. Information from such measurements can be used as input for pseudopotential band calculations or those based on the $\mathrm{k} \cdot \mathrm{p}$ approximation. The problem of constructing potentials which plague first principle band calculations is thereby avoided. Since the secular equations for such problems are generally smaller, they can be solved at sufficiently large numbers of points in the Brillouin zone to obtain the state density.

The other point concerns another recent development. A fact that has been distressing to many theorists is that, while in semiconductor calculations of optical coefficients there was always good agreement between theory and experiment in regard to both the position and magnitude of the observed structure, this has not been the case in metals. For the case of $\mathrm{Al}$ we saw quite convincing evidence that such discrepancies are on the point of disappearing, largely as a result of better calculations which deal more adequately with the k-dependence of the momentum matrix element. Indeed, other recent investigations have shown that electron-electron scattering effects, which lead to vertex corrections that might be expected to be stronger in metals than semiconductors, are, in fact, very weak in this material [3]. Even though the so-called Mayer-El Naby resonance is probably no longer with us [4], our understanding of the alkali metals is unfortunately still not in as good a shape.

It is regrettable that relatively few papers presented at the conference attempted to provide a detailed com- parison between the results obtained by different types of experiments. There was only one noteworthy exception, which was concerned with efforts to confront Knight shift data with those of soft x-ray emission experiments. There is a real need for more such detailed comparisons, even on the basis of band theory alone.

According to a paper count, superconductors and semiconductors received less attention than the simple, transition, and rare earth metals. However, there are good reasons for mentioning them even in this broad summary. As appropriate, superconductivity was not discussed as a phenomenon, but rather as a tool to extract information relevant to the state density. On the positive side, we heard how strong coupling theory can be used together with other measurements to obtain the electron-phonon coupling constants, and how measurements of the critical field at very low temperatures can be made to yield the electronic specific heat as a function of pressure with high accuracy. On the negative side, it was pointed out in connection with a general review of the information provided by tunneling experiments, that such measurements for superconductors do not really tell us all that much about the normal state properties of metals and semiconductors.

It is clear from the exquisitely detailed interpretable information being currently obtained from cyclotron resonance, magneto-optical, and even nonlinear optical data in simple semiconductors and semimetals, that our understanding of these materials is still in a somewhat more mature state than that of most metals. Of course this applies only to the classical and long studied materials like $\mathrm{Ge}$ and $\mathrm{InSb}$ and not to amorphous semiconductors which were discussed in only a single paper. However, the very beautiful interplay and agreement between theory and experiment must still be regarded as serving as a standard of excellence which solid state physics in general must continue to emulate.

Surfaces also were not discussed extensively here, largely, I think, because the theoretical ideas and techniques for dealing with such problems in realistic systems are only beginning to be developed. However, some very promising experimental techniques, notably ion neutralization spectroscopy and resonance tunneling which shed light on the nature of surfaces and important phenomena like chemisorption were described. This area will surely see a great deal of activity in the near future.

Since the discussion of a large variety of alloy systems occupied so much of the conference, let me conclude this summary with some remarks concerning this subject. We were exposed to a wide variety of data concerning many alloy systems, most of them involving 
transition metals. Certainly there are many more than were considered at the Paris Conference. However, this comparison is somewhat unfair since even now, with one or two notable exceptions which were presented here, there is still a dearth of optical information concerning disordered alloys. This is to be contrasted with the situation involving specific heat and transport measurements about which we heard a great deal. Indeed, most of the papers dealing with the electronic specific heat were concerned with alloy systems. A most interesting effect that was described dealt with the recently discovered magnetic clusters in $\mathrm{NiCu}$ alloys which can make their own appreciable contribution to the specific heat. This conjecture is quite new and deserves detailed theoretical treatment. There was also an intriguing discussion concerning rare earth metals which raised the question as to whether the $f$ electrons could possibly be at least partially itinerant in some of these systems.

One fairly obvious thing that needs emphasis in connection with these papers and that was stressed in a number of them is the need for data of single crystal specimens having known phases, and how crucial it is to avoid samples involving mixture of phases. Without these precautions, the overanxious theorists will, as they did in the case of the Mayer-El Naby anomalies, find themselves in the awkward position of explaining what Pippard already warned in 1965 might be nonfacts.

As we saw, many of the experimental techniques applicable to pure metals are relevant for disordered alloys as well. We have already mentioned optical and $\mathrm{x}$ ray data in this connection. The fruitful and relatively easily interpretable Fermi surface experiments, alas, seem to be much more difficult for many alloy systems. However, measurements such as those involving positron annihilation which also probe the Fermi surface geometry are not restricted by such criteria. They have already been very successfully used to investigate detailed Fermi surface changes in $\mathrm{Cu}-\mathrm{Al}$ [5] and as we heard here, to brass.

A great deal of progress in this area since the Paris Conference has come along the theoretical front. Until a few years ago the only theoretical models available for describing alloy behavior involved perturbation theory, the virtual crystal, or the rigid band models. However, recently a number of rather effective techniques based on scattering theory have been adapted to this problem and implemented by calculations for both model and realistic systems. These all transcend the earlier, more limited approaches. The first incisive contributions to electronic theory, made by Edwards and Beeby [6], in- volved the so-called average t-matrix approach which Soven [7] applied to brass. An extension of such calculations was discussed here. Subsequently, Soven [8] formulated a more general self-consistent effective field approach that he termed the coherent potential approximation which is more general than the other. While this was mentioned in several of the papers, it is perhaps worthy of some additional commentary because of its possible applicability to realistic alloy systems.

In this approximation the alloy is replaced by an effective medium described by a single particle non-Hermitian and complex Hamiltonian which, however, is still periodic in the case of substitutional alloys. The self-consistency condition determining this Hamiltonian is simply that an effective electron wave travelling through the crystal which impinges on an atomic site suffers no further scattering due to the random character of the crystal potential. Put another way, the effective wave, just like a Bloch wave in a crystal, is not scattered by the atoms. However, unlike the Bloch wave, the effective wave may be damped as it propagates through the crystal. The present limitation of this description is that it is only applicable to certain classes of Hamiltonians in which the random character is cell localized. The theory has the virtue of correctly reducing to the known results for small impurity concentrations and arbitrary scattering strengths on the one hand, and for arbitrary concentrations but small scattering strengths on the other. It interpolates in a physically reasonable way between these limits, yielding results that are valid for arbitrary alloy concentrations and reasonably strong scattering strengths. It fails in predicting band tailing effects, experimental evidence for which we heard described here in connection with semiconductor tunneling experiments. Also, it does not yield strictly localized states except in the limit of very small impurity concentrations.

We should note parenthetically that while the existence of such states near band edges is generally believed, some questions were raised here as to whether or not such states can exist in the middle of a tight binding band and whether or not the frequently made hypothesis that there exists a sharp demarcation between localized and nonlocalized states is correct. As we were reminded, localized states such as those due to f-electrons, can exist even in periodic systems when the Coulomb interaction is sufficiently strong. As was shown, conventional band descriptions break down under these circumstances.

To obtain the effects omitted by the coherent potential description of disordered alloys it is necessary to 
allow for the possibility of statistical clustering effects. This is a much more difficult problem. But as we heard in two of the papers presented here, some very promising progress is beginning to be made in these directions. Indeed, the early work of I. M. Lifshitz [9] has already given us an indication of the sorts of results to be expected.

Because of the previously stated limitations, the coherent potential theory in its present form is strictly speaking applicable only to isoelectronic alloys like $\mathrm{GeSi}$, where the random part of the potential is substantially confined to the core region at each site, or to $3 \mathrm{~d}$ transition-noble metal alloys in which the d-states that are most affected by the disorder are substantially localized.

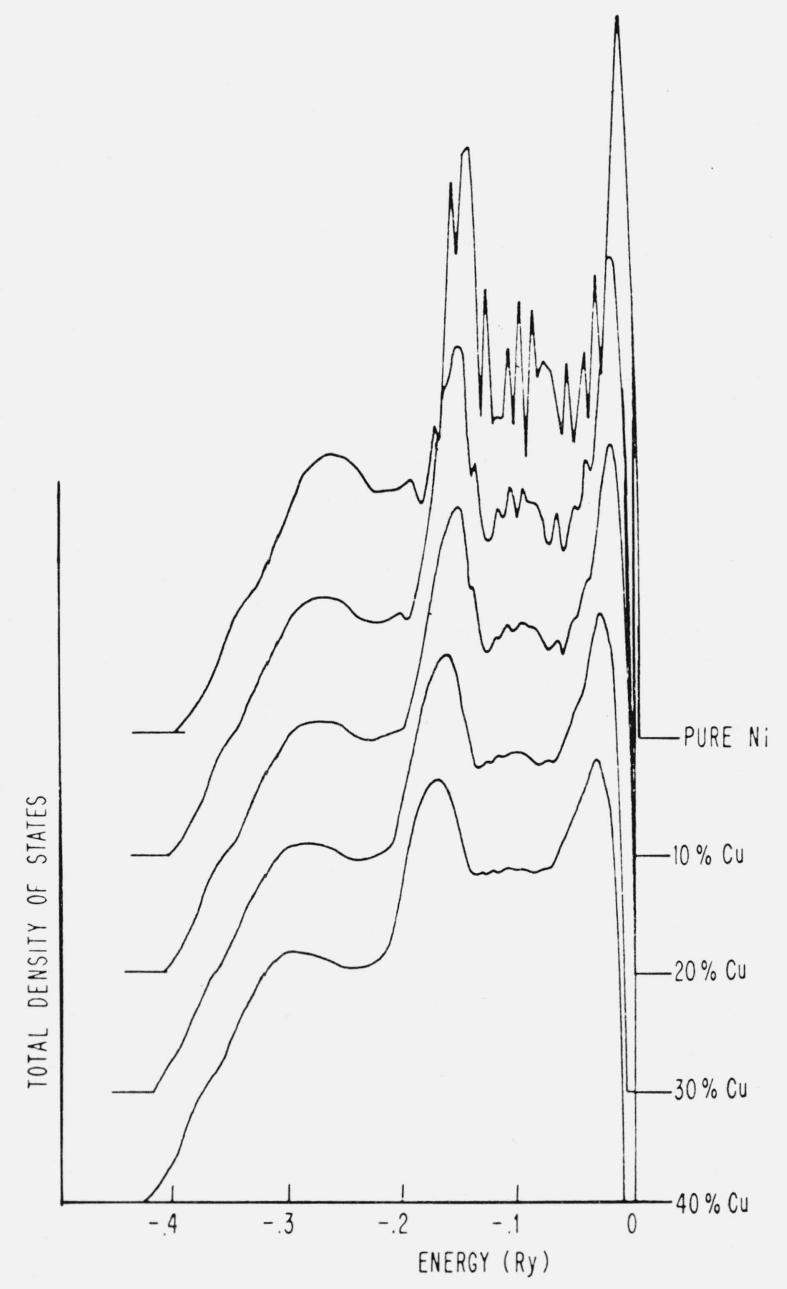

CPA CALCULATION

FOR NiCU
To give these remarks a sharper focus, I should like to show you by means of one example the results of an application of this theory to $\mathrm{CuNi}$ alloys. Figure $1 \mathrm{ex}$ hibits S. Kirkpatrick's calculations [10] for the density of states of these alloys and also the results of photoemission experiments by Seib and Spicer [11], which, for reasons already mentioned, should only be compared qualitatively with the theoretical results. It should be emphasized that these calculations do involve approximations, most of them probably not too serious. Since it would be inappropriate to discuss these in the present context, I would like to confine my remarks to a few brief comments. The first is that the coherent potential approximation is evidently applicable to quite complicated density of states functions

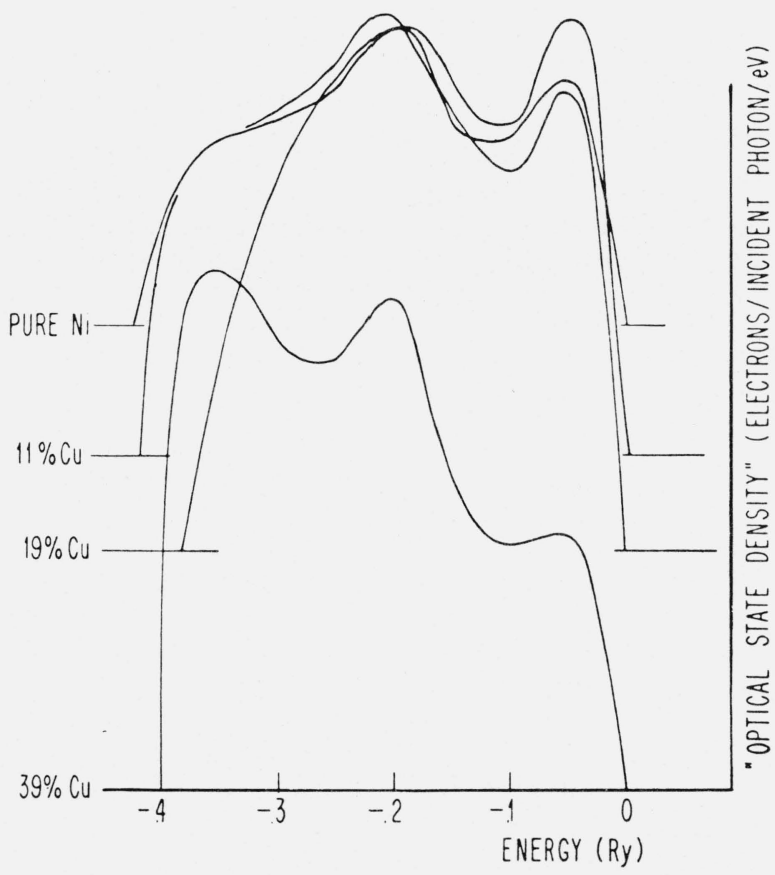

PHOTO EMISSION RESULTS FOR NICU (Seib and Spicer)

Figure 1. The hybridized $d$ state densities, calculated in the coherent potential approximation, are compared with the optical state density obtained from photoemission experiments $[10,11]$. 
which include degenerate $\mathrm{d}$-bands and hybridization with conduction bands. Second, the only input information needed for nickel-rich alloys is the hybridized nickel state density, the positions of the resonant $\mathrm{Ni}$ and $\mathrm{Cu}$ d-levels which give rise to the d-bands, and the concentration. Third, the distortion in the state density curve with increased alloying shows the rigid band model, which has been particularly popular for this alloy system, does not really apply. This is seen even more clearly in the results of calculations of the magnetic properties [12]. Finally, the prominent calculated structures and their behavior is qualitatively in accord with the experimental observations. The principal peaks remain stationary, but they change in intensity and shape in both the calculations as well as the experiment. The contribution of copper to the state density turns out to be broad and relatively structureless and comes principally from the lower regions of the d-band.

While this kind of theory predicts a wealth of detail, its quantitative validity remains an open question that must be explored further. More important, some of its present limitations must be overcome to render it applicable to a wider class of alloys. However, given the improving theoretical and experimental situation that is clearly evidenced from this conference, it seems clear that at the next meeting of this type we will surely hear about further, more extensive developments which will place the theory of alloys on a firmer footing.

After these somewhat discursive concluding remarks, let me close the conference by once again thanking everyone; organizers, speakers, and rapporteurs, questioners, commenters, and listeners for having made it as stimulating as it turned out to be.

\section{References}

[1] The Proceedings of that Conference were edited by F. Abeles and published under that title by North-Holland Publishing Company (Amsterdam) in 1966.

[2] Feldman, D. W., Parker, J. H., and Ashkin, M., Phys. Rev. Letters 21, 607 (1968).

[3] Beeferman, L., and Ehrenreich, H., (Phys. Rev., to be published).

[4] Mayer, H., and El Naby, M. H., Z. Physik 174, 289 (1963); Smith, N. V., Phys. Rev. Letters 21, 96 (1968).

[5] Fujiwara, K., Sueoka, O., and Imura, T., J. Phys. Soc. Japan 24, 467 (1968).

[6] Beeby, J. L., Proc. Roy. Soc. (London) A279, 82 (1964) and cited papers.

[7] Soven, P., Phys. Rev. 151, 539 (1966).

[8] Soven, P., Phys. Rev. 156, 809 (1967).

[9] Lifshitz, I. M., Soviet Physics - Uspekhi 7, 549 (1965).

[10] Kirkpatrick, S., Velicky, B., and Ehrenreich, H. (to be published).

[11] Seib, D. H., and Spicer, W. E., Phys. Rev. Letters 22, 711 (1969).

[12] Kirkpatrick, S., Velicky, B., Lang, N. D., and Ehrenreich, H., J. Appl. Phys. 40, 1283 (1969). 